General Mathematics Vol. 27, No. 1 (2019), 103

DOI:10.2478/gm-2019-0009

Sciendo

\title{
Book Review: Recent Advances in Constructive Approximation Theory \\ By: V. Gupta, T.M. Rassias, P.N. Agrawal, A.M. Acu Springer Optim. Appl. 138, Springer, Cham, 2018, DOI 10.1007/978-3-319-92165-5. ${ }^{1}$
}

\author{
Neha Malik
}

\section{Book Review}

This is a fine book that presents an eclectic selection of mathematical concepts and notions, which are chronologically organized. Recent research work in the area of theory of approximation is depicted in this monograph, collaboratively written by eminent mathematicians. The book gives an overview of approximation results on positive linear operators. It includes many mathematical proof techniques and ideas, which may serve as one of the most effective reference books, especially for those working in areas like post-quantum calculus, bivariate operators of discrete and integral type, estimation of differences of positive linear operators, generalized Boolean sum operators of discrete as well as continuous type and so on. It demonstrates a great influence in constructive approximation theory by intensively including computational aspects of the moduli of smoothness and Grüss \& Ostrowski type inequalities. This book will be very useful for researchers interested not only in approximation theory, but also in the field of mathematical analysis.

\section{Neha Malik}

Indian Statistical Institute

Statistics and Mathematics Unit

Bangalore - 560059, India

e-mail: neha.malik_nm@yahoo.com

\footnotetext{
${ }^{1}$ Received 20 March, 2019

Accepted for publication (in revised form) 6 May, 2019
} 